Article

\title{
Focusing High-Resolution Airborne SAR with Topography Variations Using an Extended BPA Based on a Time/Frequency Rotation Principle
}

\author{
Chunhui Lin ${ }^{1}$, Shiyang Tang ${ }^{1, *}\left(\mathbb{D}\right.$, Linrang Zhang ${ }^{1}$ and Ping Guo ${ }^{2}$ \\ 1 National Lab of Radar Signal Processing, Xidian University, Xi'an 710071, China; larrylinch@163.com (C.L.); \\ lrzhang@xidian.edu.cn (L.Z.) \\ 2 College of Communication and Information Engineering, Xi'an University of Science and Technology, \\ Xi'an 710054, China; guoping@xust.edu.cn \\ * Correspondence: sytang@xidian.edu.cn; Tel.: +86-153-1992-2377
}

Received: 4 July 2018; Accepted: 10 August 2018; Published: 13 August 2018

\begin{abstract}
With the increasing requirement for resolution, the negligence of topography variations causes serious phase errors, which leads to the degradation of the focusing quality of the synthetic aperture (SAR) imagery, and geometric distortion. Hence, a precise and fast algorithm is necessary for high-resolution airborne SAR. In this paper, an extended back-projection (EBP) algorithm is proposed to compensate the phase errors caused by topography variations. Three-dimensional (3D) variation will be processed in the time-domain for high-resolution airborne SAR. Firstly, the quadratic phase error (QPE) brought by topography variations is analyzed in detail for high-resolution airborne SAR. Then, the key operation, a time-frequency rotation operation, is applied to decrease the samplings in the azimuth time-domain. Just like the time-frequency rotation of the conventional two-step approach, this key operation can compress data in an azimuth time-domain and it reduces the computational burden of the conventional back-projection algorithm, which is applied lastly in the time-domain processing. The results of the simulations validate that the proposed algorithm, including frequency-domain processing and time-domain processing can obtain good focusing performance. At the same time, it has strong practicability with a small amount of computation, compared with the conventional algorithm.
\end{abstract}

Keywords: frequency-domain processing; extended back-projection algorithm; topography variations; computational burden; high resolution

\section{Introduction}

Over the past few decades, airborne synthetic aperture radar (SAR) has been widely adopted in remote sensing areas to produce a two-dimensional (2-D) high-resolution microwave imagery of observed scenes under all-weather and all-day conditions [1-3]. The SAR technology is important in object detection, localization, and deformation monitoring [4-6]. A variety of airborne SAR systems have been applied in practical applications, such as PAMIR, CARABAS-II, and unmanned aerial vehicle (UAV) SAR. The airborne SAR systems nowadays can illuminate the observed area at different angles, based on the SAR usually being divided into side-board SAR and squint SAR [7]. As an effective sensor, high squint SAR can also provide information about the surface structure, and can increase the flexibility to obtain the imagery of the desired district within a single pass of the airplane. Many efficient algorithms have been proposed to resolve the problems, i.e., range cell migration, azimuth spectrum aliasing, and 2-D spatial-variant Doppler frequency modulation rate [8-10]. The topography of observed scenes is also usually neglected in these conventional airborne SAR algorithms, which means 
that the ground is assumed to be a flat surface [11-13]. However, the assumption of these algorithms will be invalid, especially with increasing resolution nowadays [14]. The negligence of topography variations will cause two main problems: degradation of the focusing quality of imagery, and geometric distortion. Therefore, to satisfy the increasing demand for the high-resolution of airborne SAR systems (such as millimeter-wave SAR systems), a precise focusing algorithm for high-resolution highly squinted airborne SAR is unquestionably necessary to solve problems that are caused by topography variations.

SAR image formation for high-resolution SAR basically consists of a phase-corrected integration of the raw data samples. The different processing algorithms deviate from each other in terms of how accurately and efficiently they implement the spatially adaptive summation. In principle, the algorithms can be mainly divided into two categories: frequency-domain [15-24] and time-domain algorithms [25-28]. The former ones are aimed at raising the efficiency of the processor, but they have the limitations of integration time, flight track, and topography, which restricts the application of frequency-domain algorithms. The most general frequency-domain algorithm is the range Doppler algorithm (RDA), which decouples the range and azimuth processing with the sacrifice of focusing accuracy. Frequency-domain algorithms also include the chirp scaling (CS) algorithm [15], the Omega-k algorithm [16], and their extended forms for squinted airborne SAR. They are usually based on the assumption of a strictly straight trajectory. The nonlinear chirp scaling (NLCS) algorithm [17] and its extension [18-22] are the common algorithms that are used to deal with the spatial variance, especially the azimuth variance. These frequency-domain algorithms are developed by scholars before the graphics processing unit was applied. In general, these aforementioned algorithms have not taken the topography into account. The precise topography and aperture dependent (PTA) algorithms [25] are proposed to realize the motion compensation based on block processing. To avoid discontinuities at block borders, it usually takes a $50 \%$ overlap, which results in a relatively high computational burden. The subaperture topography- and aperture- dependent (SATA) algorithm [26] is another typical algorithm that increases the accuracy of the topography variants. This uses the short time Fourier transform in subaperture along the azimuthal direction.

The typical time-domain algorithm is the time-domain back-projection (BP) algorithm, which has been widely accepted as a precise algorithm for all SAR reconstructions [29,30]. In principle, BP algorithm is a process of pulse-by-pulse and pixel-by-pixel. Thus, it can be applied in almost every SAR mode, configuration, and terrain. At the same time, the digital elevation model (DEM) has been a mature technology in the remote sensing field [31-33]. The combination of the BP algorithm and DEM can perfectly realize the topography-dependent motion compensation. Due to the pixel-by-pixel character, the biggest disadvantage of the BP algorithm is the computational burden. Usually, the computation of the time-domain BP algorithm is proportional for a desired image with, and azimuth sampling points. Compared with frequency-domain algorithms, the time-domain BP algorithm provides lower adaptability and practicability. To improve the computational efficiency for time-domain algorithms, two major approaches have been developed: parallel computing platforms and incremental modifications of the BP algorithm. Many methods have been proposed to improve the operational efficiency of the BP algorithm. The representative ones are the fast back projection (FBP) algorithm [34] and the fast factorized back projection (FFBP) algorithm [35]. The FBP algorithm and FFBP algorithm both consist of a number of processing stages, and combine the subaperture technology with polar grids processing. To a certain degree, they both reduce the computational burden, while keep most of the advantages of the original BP algorithm. The extensions of the FBP algorithm and the FFBP algorithm were applied in different SAR scenes [36-43]. However, the subaperture will introduce problems of subaperture division and sub-image mosaic [44]. Also, topography variations are taken in account, which will cause image distortion. In general, for high-resolution airborne SAR systems, it becomes very difficult for the methods mentioned above, to realize the focusing of the observed scene, with the increase in resolution, squint angle, and scene coverage. A fast focus algorithm based on full-aperture processing is necessary, especially when topography variations cannot be ignored. 
However, some overlap between the blocks is considered, to reduce the appearance of sidelobe in the SATA algorithm.

In this paper, we proposed an extended back-projection (EBP) algorithm for the squinted high-resolution airborne SAR, with the consideration of topography variations. To avoid subaperture division and image mosaic, the proposed algorithm will be based on full-aperture processing, rather than partitioned processing. The range domain data or azimuth domain data is processed as a bulk. This proposed algorithm applies a time-frequency rotation operation, which is similar to the two-steps approach in certain extent. Compared with the conventional time-domain BP algorithm, the proposed EBP algorithm needs lesser amounts of computation. At the same time, it remains high-resolution performance for high-resolution airborne SAR systems with topography variations.

The paper is organized as follows. Section 2 is dedicated to the model of high-resolution airborne SAR with topography variations and analysis of phase errors without consideration, and the derivation of corresponding expressions are given. The details of the proposed EBP algorithm, i.e., the frequency-domain processing and time-domain processing, are explained in Section 3. Then, several simulation results of the performance of the proposed algorithm are provided in Section 4 . Section 5 discusses the problems about parameter selection and the computational burden for the proposed EBP algorithm. The final conclusion is given in Section 6.

\section{Modeling and Analysis}

\subsection{Modeling of Airborne SAR}

Traditionally, the movement of airborne SAR is assumed as a linear uniform motion in a straight line, with the ground scene being flat. It was reasonable for the short integration time, low-resolution airborne SAR. The hyperbolic range model, neglecting the impacts of topography variations on the imaging results, would introduce significant phase errors with the increasing resolution for airborne SAR systems. During the acquisition interval, neither the motion error effects, nor the topography variations could be ignored. A general geometry of high-resolution airborne SAR is shown in Figure 1.

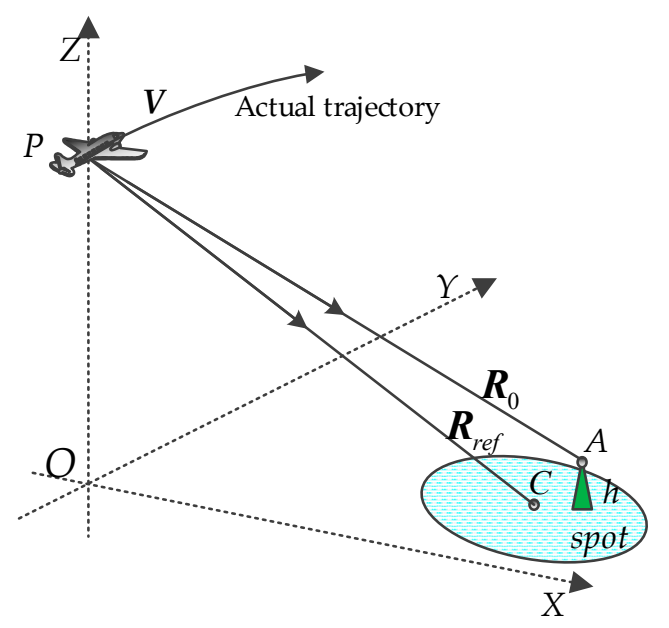

Figure 1. Geometrical model of high-resolution airborne synthetic aperture (SAR).

It is noted that point $P$ is the reference position of the fight path at the aperture center moment (ACM), and points $C$ and $A$ are respectively the reference and arbitrary targets on the ground scene, $h$ is the height of the point $A, \boldsymbol{R}_{r e f}$ and $\boldsymbol{R}_{0}$ are respectively the slant range vectors from $P$ to $C$, and $P$ to $A$ at $\mathrm{ACM}$, and $V$ is the velocity vector of the platform. With reference to the imaging geometry, the slant range history of point $A$ is expressed as:

$$
r(\eta ; A)=r_{i}(\eta ; A)+r_{e}(\eta ; A)+r_{t}(\eta ; A)
$$


where $\eta$ is the slow time, $r_{i}(\eta ; A), r_{e}(\eta ; A)$, and $r_{t}(\eta ; A)$ are respectively the range history, motion errors, and the topography variations of $\operatorname{target} A$, and $r_{i}(\eta ; A)$ are expressed as:

$$
r_{i}(\eta ; A)=\left|\boldsymbol{R}_{0}-\boldsymbol{V} \cdot \eta\right|
$$

where $|\cdot|$ denotes the norm operation. In this general model, $r_{e}(\eta ; A)$ and $r_{t}(\eta ; A)$ cannot be ignored in the case of high-resolution.

According to Equation (1), the received echo of point $A$ is expressed as:

$$
\begin{aligned}
S_{0}\left(t_{r}, \eta\right)= & \varepsilon_{0} \omega_{r}\left(t_{r}-2 r(\eta ; A) / c\right) \omega_{a}\left(\eta-\eta_{0}\right) \\
& \cdot \exp \left(-j 4 \pi f_{c} r(\eta ; A) / c\right) \cdot \exp \left(j \pi \gamma\left[t_{r}-2 r(\eta ; A) / c\right]^{2}\right)
\end{aligned}
$$

where $t_{r}$ is the range fast time, $f_{c}$ and $\gamma$ are the carrier frequency and the frequency modulation (FM) rate of the transmitted signal, respectively, $\varepsilon_{0}$ is the complex scattering coefficient, $\omega_{r}(\cdot)$ and $\omega_{a}(\cdot)$ are respectively the range and azimuth envelopes in the time-domain, and $\eta_{0}$ denotes the Doppler center time of the point target $[45,46]$.

\subsection{Problem}

In conventional SAR imaging processing, the phase error brought about by topography variation was not considered, which would greatly deteriorate the final image and limit the ground scene size. In this subsection, the quadratic phase error (QPE) brought by topography variation $r_{t}(\eta ; A)$ was analyzed for high-resolution airborne SAR, according to Equations (1) and (2). Utilizing the parameters listed in Table 1, Figure 2 shows the absolute QPEs with different variables, i.e., resolution, squint angle, and scene size. The unit of the figures was $\pi$. Figure $2 a, b$ is respectively, the simulation results in the range/height and azimuth/height domains with azimuth resolution and the squinted angle, respectively being $0.25 \mathrm{~m}$ and $60^{\circ}$. Figure $2 \mathrm{c}, \mathrm{d}$ is the simulation results in the resolution/height and squint angle/height domains, respectively. Clearly, the maximum QPE in each domain was far larger than $\pi / 4$, which could not be accepted in the high-resolution airborne SAR imaging. Moreover, the QPE increased with the scene coverage, azimuth resolutions, and the squint angles, as shown in Figure 2. It was concluded that the effects that were brought by topography variations cannot be ignored in the case of high-resolution, large-swath, and high squint angles. Figure 3 shows the absolute position errors (PEs) that were introduced by topography variations. The unit of the figures was impulse-response width (IRW). It was seen that the PEs were all generally larger than one IRW in each height cell, and that they would greatly distort the final focused image.

Table 1. Parameter settings.

\begin{tabular}{cc}
\hline Parameters & Value \\
\hline Carrier frequency & $9.65 \mathrm{GHz}$ \\
Pulse duration & $2.5 \mu \mathrm{s}$ \\
Pulse Bandwidth & $300 \mathrm{MHz}$ \\
Sampling frequency & $420 \mathrm{MHz}$ \\
Reference slant range & $25.0 \mathrm{~km}$ \\
Altitude & $8.0 \mathrm{~km}$ \\
Velocity vector & $(0,120,0) \mathrm{m} / \mathrm{s}$ \\
\hline
\end{tabular}

Simulation results provide a visually view of the impact brought by topography variations. It is noted that the large QPEs and PEs were eliminated for high-resolution airborne SAR imaging. The focusing approach was a rigorous one in this work, compared with those for the conventional cases which assume that the ground scene is flat. 


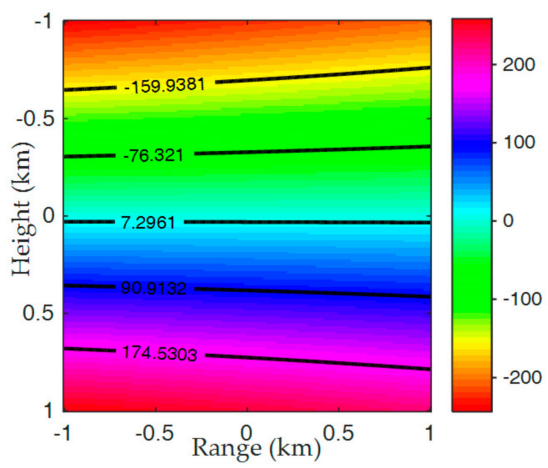

(a)

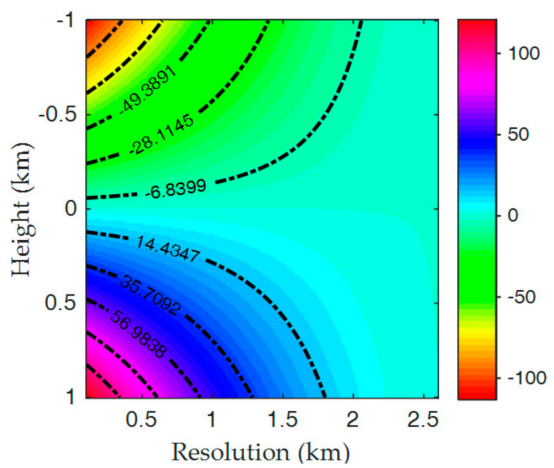

(c)

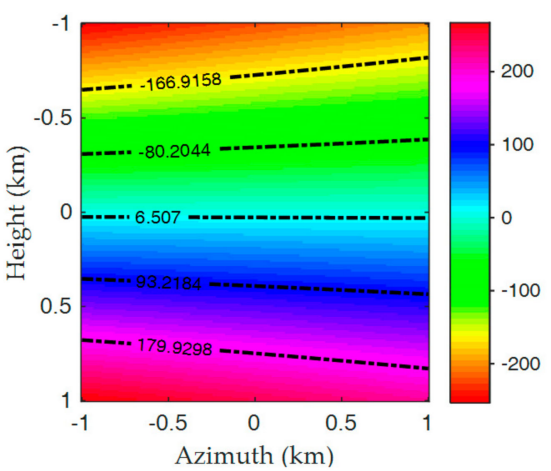

(b)

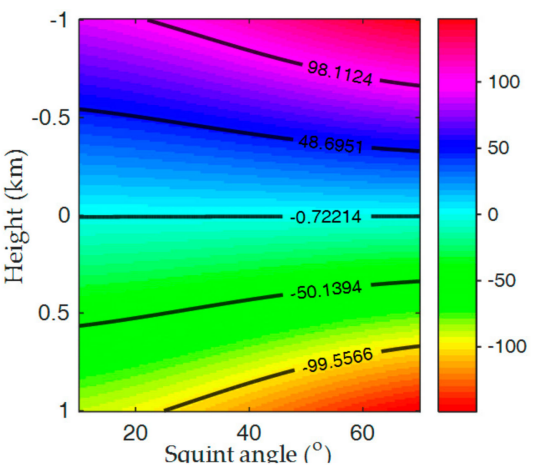

(d)

Figure 2. Quadratic phase errors (QPEs) introduced by topography variations in different domains: (a) QPEs introduced by topography variations in range/height domain; (b) QPEs introduced by topography variations in azimuth/height domain; (c) QPEs introduced by topography variations in the resolution/height domain; (d) QPEs introduced by topography variations in the squint angle/height domain.

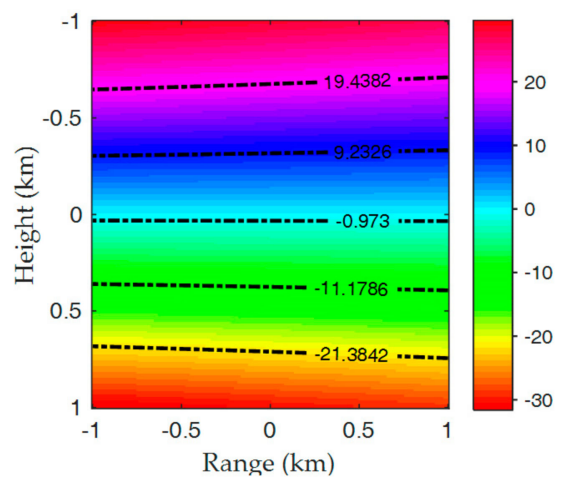

(a)

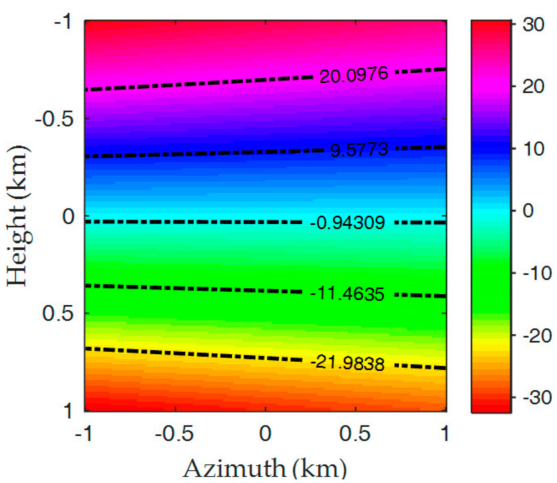

(b)

Figure 3. PEs introduced by topography variations in different domains: (a) PEs introduced by topography variations in the range/height domain; (b) PEs introduced by topography variations in the azimuth/height domain.

For the high-resolution airborne SAR, two main aspects should be considered:

(1) Three-dimensional (3-D) variations in time-domain. Clearly, the general range history in Equation (1) was a vector variable function, which means that one should consider the changes in range, azimuth, and height directions, when designing the focusing approach in the time-domain. An accurate equation indicates the good performance of the proposed focusing method, or it 
would lead to a great deterioration in the final imaging result, including the IRW, the peak side-lobe ratio (PSLR), and the integration side-lobe ratio (ISLR).

(2) 3-D variations in the frequency-domain. Because of the complex composition terms in Equation (1), it is difficult to derive the 2-D spectrum, even when using the method of the series reversion (MSR). Moreover, with the complex range history, the focused approach designed in the 2-D frequency-domain would be more complicated than those in the time-domain. Thus, the process directly applied in frequency-domain was not the best choice for an efficient algorithm design for high-resolution airborne SAR in this work.

\section{Imaging Algorithm}

For the high-resolution airborne SAR, the complex range history in Equation (1) greatly increased the difficulty in imaging, because the 3-D variations that were distributed on the ground scene were difficult to eliminate, especially in the height direction. To solve these problems, a novel focusing algorithm was presented in this section. The proposed EBP algorithm took into account the topography variations, and had the advantages of accuracy and efficiency. The imaging algorithm could be divided into two steps. The first step, i.e., the frequency-domain processing, was used to greatly decrease the virtual aperture length by applying the equivalent time-frequency rotation operations in the frequency-domain. The second step, i.e., the time-domain processing, was to remove the 3-D spatial variations, including the 2-D spatial variations in the horizontal plane, and the spatial variation was brought by topography variations in the vertical plane by performing BP in the time-domain. The specific implementation of these two parts: frequency-domain processing and time-domain processing, was presented as following.

\subsection{Frequency-Domain Processing}

The defect of the conventional BP algorithm was poor computational efficiency. The reduction of the computational burden would be the key point to improving the imaging efficiency. In this section, a frequency-domain approach was presented to compress the data in an azimuth time-domain for the $\mathrm{BP}$ algorithm. The essential of the frequency-domain processing in this work was an equivalent time-frequency rotation operation to change the support area (aperture length) in the azimuth time-domain, which was similar to that of the traditional two-step approach proposed in [47-49]. The difference was that the traditional two-step approach was used to change the equivalent azimuth bandwidth, to avoid spectrum aliasing, whereas the proposed one was to decrease the virtual aperture length, to reduce the computational burden of the BP algorithm. Moreover, they performed in different domains. For instance, the first step of the frequency-domain processing was implemented in the azimuth frequency, while one of the traditional two-step approaches was implemented in the azimuth time-domain. So we first transformed Equation (3) into the range/Doppler domain, i.e., $S_{1}\left(t_{r}, f_{\eta}\right)$, where $f_{\eta}$ was the azimuth frequency. Similar to that of the two-step approach [47], the reference function was constructed as:

$$
H_{1}\left(f_{\eta}\right)=\exp \left(-j \pi \alpha_{0} f_{\eta}^{2}\right)
$$

where $\alpha_{0}$ is the scaling factor and it determines the sampling interval in the time-domain after frequency-domain processing. Convoluting the range/Doppler signal $S_{1}\left(t_{r}, f_{\eta}\right)$ of point $A$ with the reference function expressed in Equation (4) yields:

$$
\begin{aligned}
S_{2}\left(f_{\eta}^{\prime}\right) & =S_{1}\left(t_{r}, f_{\eta}^{\prime}\right) \otimes_{a z} H_{1}\left(f_{\eta}^{\prime}\right) \\
& =\frac{\exp \left(-j \pi \alpha_{0} f_{\eta}^{\prime 2}\right)}{\text { Residual Phase }} \cdot \frac{\int S_{1}\left(t_{r}, f_{\eta}\right) \exp \left(-j \pi \alpha_{0} f_{\eta}^{2}\right)}{\text { deramping }} \cdot \frac{\exp \left(j 2 \pi \alpha_{0} f_{\eta} f_{\eta}^{\prime}\right)}{\text { IFT Kernel }} d f_{\eta}
\end{aligned}
$$

where $\otimes_{a z}$ denotes the azimuth convolution in the frequency-domain. It is clearly that the convolution can be achieved by three operations, i.e., the deramping operation, the inverse Fourier transform 
(IFT) with new IFT kernel, and the residual phase compensation, as marked in Equation (5). The frequency-domain processing had the same principle as that of the azimuth preprocessing [49] that was applied in the time-domain, which could be regarded as the 'time-frequency rotation' [48]. According to the new IFT kernel, one can obtain that:

$$
\left\{\begin{array}{l}
\eta^{\prime}=\alpha_{0} f_{\eta} \\
\eta=\alpha_{0} f_{\eta}^{\prime}
\end{array}\right.
$$

where $\eta^{\prime}$ and $f_{\eta}^{\prime}$ are the new azimuth time and frequency variables, respectively. The residual phase compensation was expressed as:

$$
H_{2}\left(f_{\eta}^{\prime}\right)=\exp \left\{-j \pi \alpha_{0} f_{\eta}^{\prime 2}\right\}
$$

The convolution in Equation (5) performed in the frequency-domain could be rewritten as a product in the time-domain, i.e.,:

$$
S_{2}\left(t_{r}, \eta^{\prime}\right)=S_{1}\left(t_{r}, \eta^{\prime}\right) \cdot H_{1}\left(\eta^{\prime}\right)
$$

where $H_{1}\left(\eta^{\prime}\right)$ is the time-domain form of $H_{1}\left(f_{\eta}^{\prime}\right)$, and $S_{2}\left(t_{r}, \eta^{\prime}\right)$ is the form of $S_{2}\left(f_{\eta}^{\prime}\right)$ in the 2-D time domain. To acquire $S_{2}\left(t_{r}, \eta^{\prime}\right)$, one needs to compensate the second term on the right side of Equation (8), and the compensation function can be constructed as

$$
H_{3}\left(\eta^{\prime}\right)=\exp \left(-j \pi \eta^{\prime 2} / \alpha_{0}\right)
$$

Multiplying Equation (9) with Equation (8), one finally obtains the 2-D time-domain expression which has a form that is similar to that of the original echoes, except for different azimuth time variable $\eta^{\prime}$. According to Equation (6), it is noted that the scaling factor $\alpha_{0}$ can be selected to decrease support area (virtual aperture length) to reduce the computational burden of the time-domain processing in the next step. Thus, the frequency-domain processing was a key part in this work.

In order to explain the kernel of the frequency-domain processing, the diagram for frequencydomain processing is graphically shown in Figure 4. Four targets are distinguished in the azimuth dimension. The time-frequency diagrams (TFDs) of received raw signal and reference function $H_{1}$ are shown in Figure $4 \mathrm{a}$. As shown in Figure $4 \mathrm{~b}$, the received signal obtained the new supporting area after the deramping operation, in part of Equation (5). At this step, the echo signal is multiplied with Equation (4) in the azimuth frequency-domain. Then, an equivalent IFT operation with a new IFT kernel in the azimuth from $\eta$ and $f_{\eta}$ to $\eta^{\prime}$ and $f_{\eta}^{\prime}$ was implemented, and Figure 4c shows the results of the equivalent IFT operation. At the last step, the compensation was implemented by using Equations (7) and (8) in different domains, and the renovated echo signal was obtained, as shown in Figure $4 \mathrm{~d}$. After the operation above, the virtual aperture length of the new echo signal was smaller than the one of original echo signal. This meant that the computational burden of the following algorithm could be decreased, if the data of processed echo signal was selected properly. 


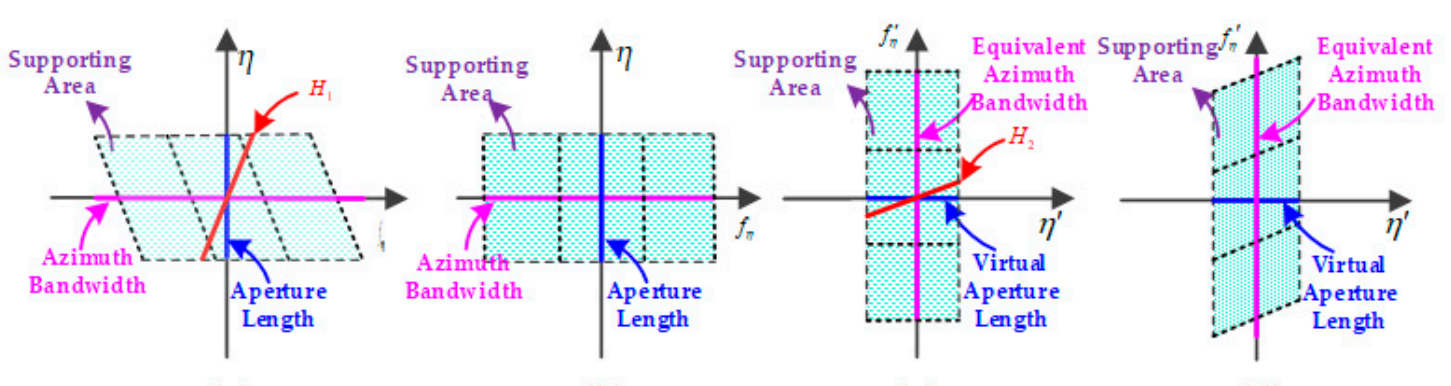

(a)

(b)

(c)

(d)

Figure 4. Steps of the frequency-domain processing using the time-frequency diagrams (TFDs). (a) Echo signal and reference function $H_{1}$ in the range/Doppler domain; (b) signal after deramping; (c) signal after inverse Fourier transform (IFT) with new IFT kernel and residual phase compensation function $\mathrm{H}_{2}$; (d) signal after the residual phase compensation.

\subsection{Time-Domain Processing}

The time-domain algorithms are mainly the back projection algorithm (BPA) and its extensions [30-35]. They implement the phase-corrected integration for each imaged point separately, by taking into account the individual propagation delay. So the application of the BPA, and its extensions can easily adapt to airborne SAR with topography variations. However, in terms of computational burden, the BPA is not always the best choice, compared with those of the frequency-domain algorithms. To avoid the heavy computational burden, the combination of the conventional BPA and the frequency-domain processing in Section 3.1 was applied in this work, to improve the efficiency of the imaging algorithm.

After the frequency-domain processing, the echo signal was transformed into the range frequency-domain as:

$$
S_{3}\left(f_{r}, \eta^{\prime}\right)=\varepsilon_{0} \omega_{r}\left(f_{r}\right) \omega_{a}\left(\eta^{\prime}\right) \exp \left(-j \pi \frac{f_{r}^{2}}{\gamma}\right) \exp \left(-j \frac{4 \pi\left(f_{c}+f_{r}\right)}{c} r\left(\eta^{\prime} ; A\right)\right)
$$

Performing the range compression, the compression factor was expressed as:

$$
H_{4}\left(f_{r}\right)=\exp \left(j \pi \frac{f_{r}^{2}}{\gamma}\right)
$$

Then, multiplying Equation (11) with Equation (10), one can get:

$$
\begin{aligned}
S_{4}\left(f_{r}, \eta^{\prime}\right) & =S_{3}\left(f_{r}, \eta^{\prime}\right) \cdot H_{4}\left(f_{r}\right) \\
& =\varepsilon_{0} \omega_{r}\left(f_{r}\right) \omega_{a}\left(\eta^{\prime}\right) \exp \left(-j \frac{4 \pi\left(f_{c}+f_{r}\right)}{c} r\left(\eta^{\prime} ; A\right)\right)
\end{aligned}
$$

Transforming Equation (12) into 2-D time-domain yields:

$$
S_{5}\left(t_{r}, \eta^{\prime}\right)=\varepsilon_{0} G_{r} G_{\eta} \operatorname{sinc}\left[\Delta f_{r}\left(t_{r}-2 r\left(\eta^{\prime} ; A\right) / c\right)\right] \exp \left(-j \frac{4 \pi}{\lambda} r\left(\eta^{\prime} ; A\right)\right)
$$

where $\Delta f_{r}$ is the bandwidth of the transmitted signal, $G_{r}$ and $G_{\eta}$ represent the range and the azimuth envelope gain in the frequency-domain respectively, and $\lambda$ is the wavelength of the transmitted signal.

Based on the new azimuth time $\eta^{\prime}$, the BPA was applied in Equation (13) to achieve the focused image. It should be noted that the sampling number in azimuth time-domain can be significantly decreased by selecting properly scaling factor $\alpha_{0}$, which can greatly reduce the computational burden with slight deterioration of imaging quality. This was the main reason that we performed the frequency-domain processing first in this work. 
Figure 5 shows the illustration of the proposed algorithm. The first step is frequency-domain processing. It was seen that both the virtual aperture length and the new sampling interval were smaller than the original ones, which was decided by the selection of $\alpha_{0}$. The length of the original aperture was reduced greatly, and the sampling interval became smaller than the original one. So, some of the positions in the flight path became redundant. In the new aperture, the green spots represented the chosen sampling points, while the red ones represented the redundant sampling points. Thus, a lesser number of positions could be selected for the BPA to reduce the computational burden, as shown in Figure 5. Then, the time-domain processing, i.e., BPA, was applied in the 2-D time-domain. It was important to note that the DEM data should be known to achieve the accurate range history.

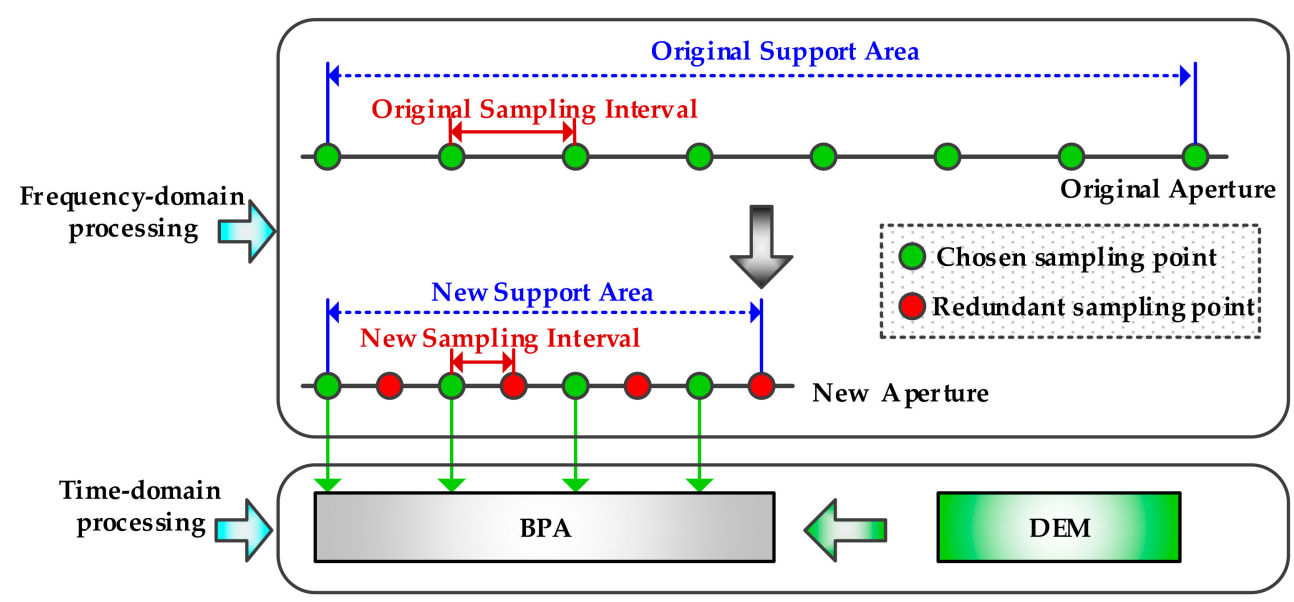

Figure 5. Illustration of proposed algorithm.

\subsection{Flowchart of the EBP Algorithm}

Based on the aforementioned discussion, Figure 6 presents the flowchart of the EBP algorithm. $H_{1}$, $\mathrm{H}_{2}, \mathrm{H}_{3}$, and $\mathrm{H}_{4}$ were respectively expressed as Equations (4), (7), (9) and (11). Clearly, the proposed EBP algorithm included two parts: frequency-domain processing was used to reduce the length of the virtual aperture of the received signal and the computational burden of the following algorithm; time-domain processing solves the problems that are caused by topography variations, with the combination of the selection of the sampling points, the DEM data, and the back-projection algorithm. The processing procedure of the proposed algorithm was practically denoted as follows:

1. Azimuth Fourier transform (FT). Apply an azimuth FT on the raw data;

2. Deramping processing; the first step of the frequency-domain processing. Modulate the raw data with Equation (4);

3. Equivalent Azimuth IFT; the second step of the frequency-domain processing. Apply the transformation in the data from the $\eta$ and $f_{\eta}$ coordinate, to the $\eta^{\prime}$ and $f_{\eta}^{\prime}$ coordinate;

4. Residual phase compensation; the final step of the frequency-domain processing. Compensate the data with Equation (7) in the new azimuth frequency-domain;

5. Azimuth IFT. Transform the data from the $f_{\eta}^{\prime}$ domain to $\eta^{\prime}$ domain;

6. Second phase compensation. Compensate the data with Equation (9) in the new azimuth time-domain;

7. Range FT. Apply a range of FT on the renovated data. Then, the range compression will be implemented in the range frequency-domain;

8. Range compression. Compress the data in the range frequency-domain with Equation (11);

9. Range IFT. Transform the data from the $t_{r}$ domain to $f_{r}$ domain; 
10. Selection of samples. The important sampling points are selected appropriately, and the premise is that the echo signal will not alias the azimuth frequency-domain. In this way, the computational burden of the BP algorithm will be reduced;

11. BP processing. Calculate the accurate slant range from each azimuth position to the targets, with the combination of the airborne acquisition scenarios and the DEM data. The other substeps in this step are similar to those of the classical BP processing.

For the purpose of simplicity and clarity, the work mode of the high-resolution airborne SAR system is designed as a spotlight SAR in the previous discussion of the proposed algorithm. Meanwhile, it is assumed that the airborne SAR flies along the linear trajectory. Actually, the proposed EBP algorithm can be also applied in other work modes, such as the stripmap SAR, terrain observation by progressive scans (TOPS) SAR, or the sliding spotlight SAR. These modes are distinguished, based on the variation of the antenna beam direction, and they are all called beam steering SAR for simplicity [50]. It is well known that the time-domain back-projection algorithm can be considered as a linear transformation from radar echo data to the SAR scene; thus, the proposed algorithm based on the BP algorithm could be applied in SAR, with the above-mentioned modes. Furthermore, the EBP algorithm was also applicable to the other SAR system (including the airborne SAR and spaceborne SAR), flying along the curve trajectory.

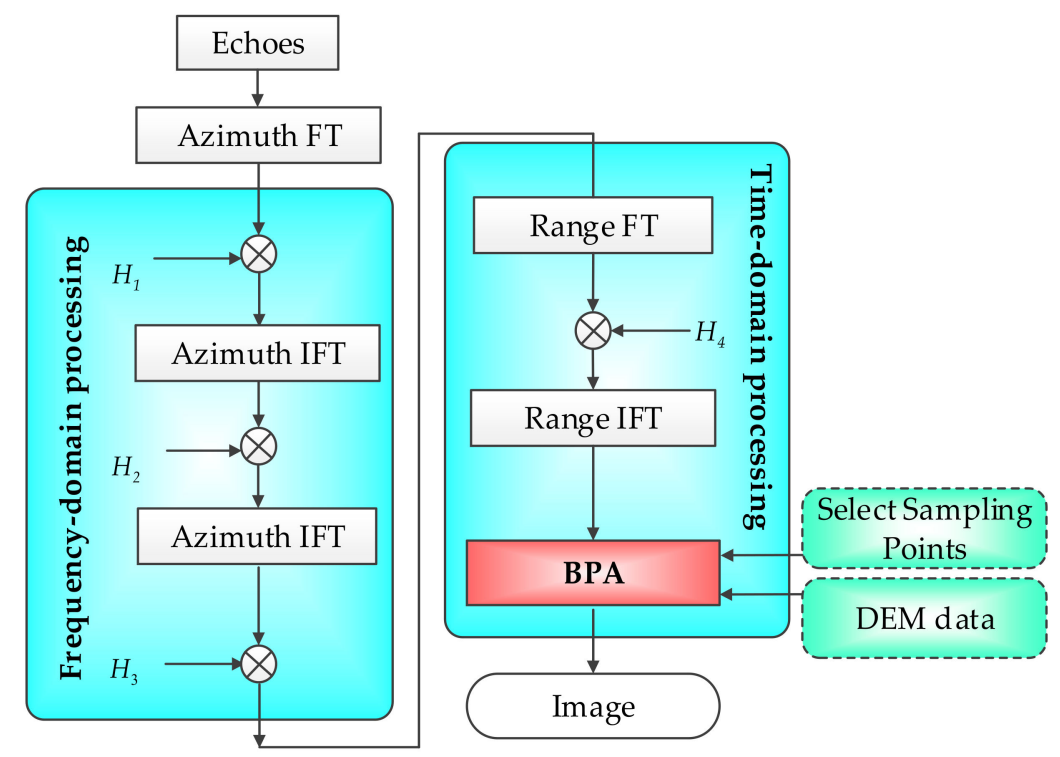

Figure 6. The flowchart of the EBP algorithm. In this flowchart, frequency-domain processing and time-domain processing are included.

\section{Simulation Results}

In this section, to prove the efficiency of EBP algorithm, spot matrix simulation results and SAR scene simulation results are respectively presented.

\subsection{Spot Matrix Simulation Results}

In this subsection, the performance of the proposed EBP algorithm is evaluated in the cases without, and with consideration of the motion errors. The parameters for the simulation are listed in Table 1.

Figure 7 is the distribution of the targets in the illuminated spot matrix. The scene size (range $\times$ azimuth) is about $1.2 \mathrm{~km} \times 1.2 \mathrm{~km}$. The targets in the different location of the scene was at different altitudes. 


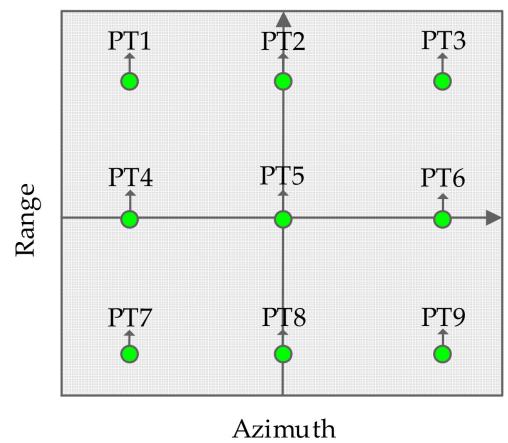

Figure 7. Distribution of targets in the illuminated spot matrix which contained nine-point targets.

\section{Case I}

In this experiment, simulation results without considering the topography variations were used for comparisons. The airborne SAR worked in the spotlight mode. The motion errors were not added in this case. The heights of targets PT1 and PT9 were respectively set as -120.0 and $180.0 \mathrm{~m}$, with respect to the reference point PT5, the squint angle was $30^{\circ}$, and the azimuth resolution was $0.681 \mathrm{~m}$. The traditional chirp scaling algorithm (CSA) (e.g., [15]) was used for comparison.

Figure 8 shows the comparative results by the proposed algorithm and CSA. From Figure 8 , we see that the topography variations in the vertical direction were greatly decreased, and the targets with different range and azimuth positions by the proposed approach were visually well-focused. However, for the CSA without considering the topography variations in the vertical direction, although the reference target in the scene center could be well-focused, the targets on the edges remained highly defocused for the reason, that the errors introduced by the target heights cannot be ignored in the high-resolution case.
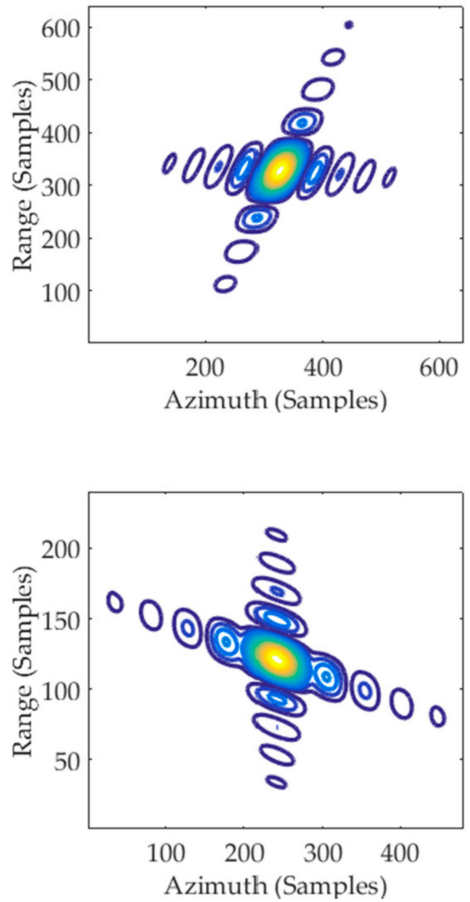

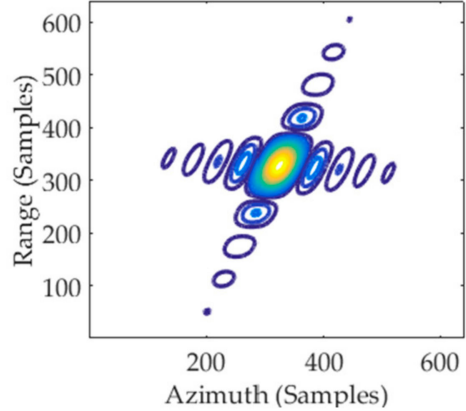

(a)

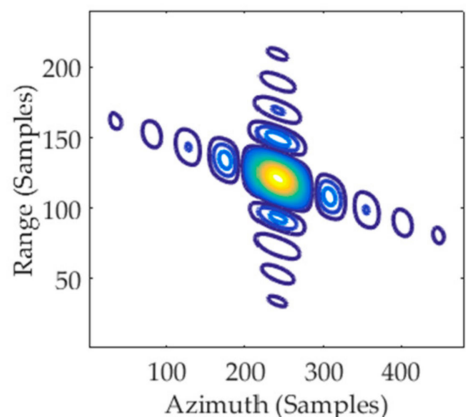

(b)
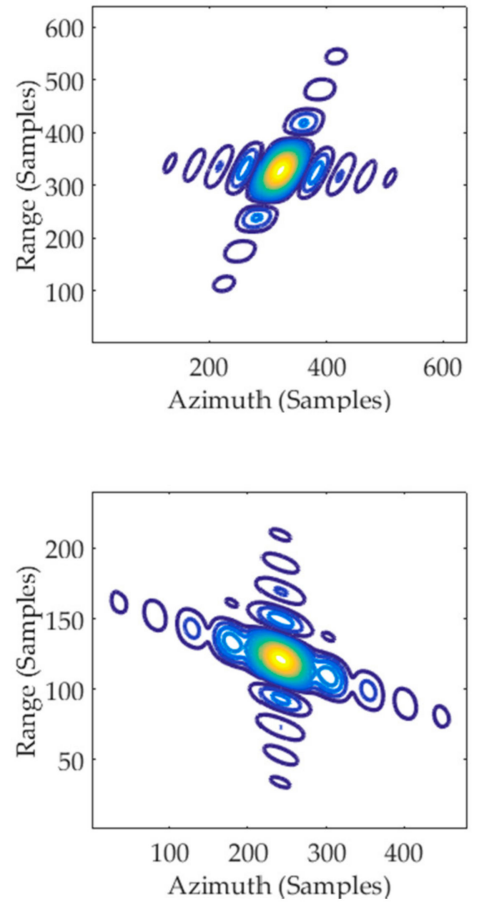

Figure 8. Comparative results by proposed algorithm and CSA. (Left to right) Targets PT1, PT5, and PT9, respectively. (a) Contour plot of targets (PT1, PT5, and PT9) processed by the EBP algorithm; (b) Contour plot of targets (PT1, PT5, and PT9) processed by CSA. 
To acquire the further comparison of the focusing performance, a quantitative analysis with IRW, PSLR, and ISLR was used as criteria. The corresponding imaging performances of the targets PT1, PT5, and PT9 are calculated, and the results of proposed algorithm, and the CSA were listed in Table 2. Both the contour results and imaging quality parameters of the proposed algorithm approach the theoretical values of IRW $(0.681 \mathrm{~m})$, PSLR $(-13.26 \mathrm{~dB})$, and ISLR $(-9.8 \mathrm{~dB})$, which indicated that the proposed algorithm could be well-applied to the high-resolution airborne SAR with topography variations. On the other hand, the corresponding parameters that were obtained by the CSA were relatively worse, especially for the targets in the edge of the scene, which were much less than the theoretical values. It means that the CSA was inferior to the proposed algorithm.

Table 2. Imaging quality parameters in Simulation Case I.

\begin{tabular}{ccccc}
\hline Method & Target & IRW (m) & PSLR (dB) & ISLR (dB) \\
\hline \multirow{3}{*}{ Proposed algorithm } & PT1 & 0.687 & -13.22 & -10.03 \\
& PT5 & 0.681 & -13.27 & -10.05 \\
& PT9 & 0.679 & -13.24 & -10.02 \\
\hline \multirow{2}{*}{ CSA } & PT1 & 0.795 & -7.23 & -6.65 \\
& PT5 & 0.682 & -13.25 & -10.06 \\
& PT9 & 0.813 & -7.14 & -6.37 \\
\hline
\end{tabular}

Case II

In this experiment, the heights of targets PT1 and PT9 were respectively 452.5 and $-231.1 \mathrm{~m}$, with respect to target PT5, which was set as the reference point in Figure 7 . The squint angle was $60^{\circ}$, and azimuth resolution was $0.472 \mathrm{~m}$. The PTA-MoComp approach (PMA) [25] was performed for comparisons. Figure $9 \mathrm{a}$ is the motion errors extracted from the airborne inertial navigation system. Figure $9 \mathrm{~b}$ shows the focused result of the simulation spot matrix. It is well-noted that the positions of targets PT1 and PT9 were well-located without distortions in the image.

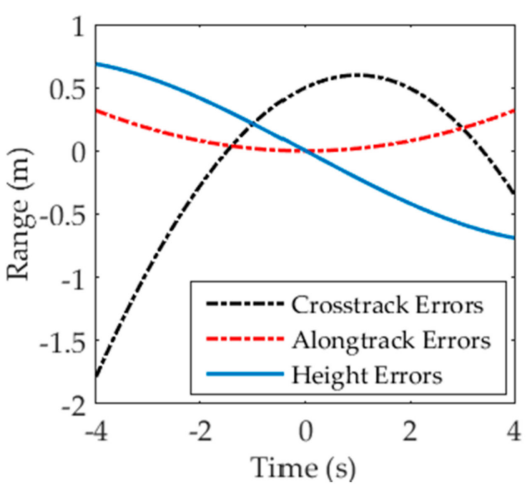

(a)

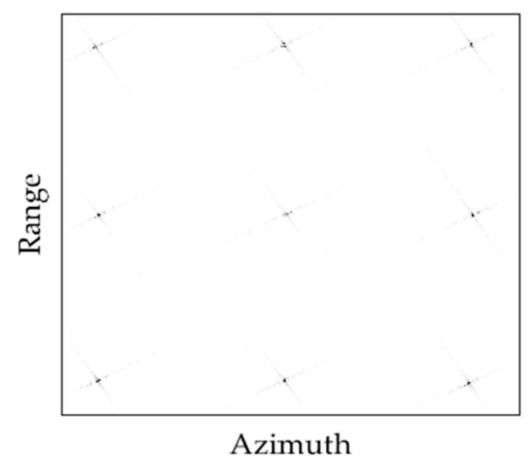

(b)

Figure 9. Motion errors and simulation results: (a) motion errors extracted from the airborne inertial navigation system; (b) focused result of the simulation spot matrix by the proposed algorithm.

Figure 10 shows the comparative results of the azimuth profiles focused by PMA and the proposed algorithm. It was well-noted that the targets were visually well-focused, with a relatively clear separation of the main lobes and the first and subsequential sidelobe, by the proposed algorithm. Since there was no extra windowing operation, the theoretical values of PSLR were about $-13 \mathrm{~dB}$. Clearly, the proposed algorithm had a good performance (red dashed lines in figures). For the traditional method of PMA, the reference target could be well focused; however, the edge targets had great degradations because the post azimuth-matched filter was sensitive to azimuth-dependent motion errors. The imaging quality parameters were listed in Table 3. Imaging quality parameters of the 
proposed algorithm were obviously close to the theoretical values of IRW (0.472 m), PSLR ( $-13.26 \mathrm{~dB})$, and ISLR (-9.8 dB), while the ones of the PMA were relatively worse. Our algorithm was noted to be generally superior to the PMA. Consequently, with the incorporation of the frequency-domain processing into the time-domain processing, promising results were obtained for high-resolution airborne SAR.

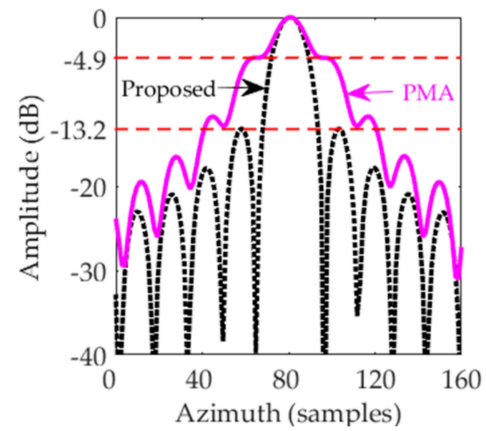

(a)

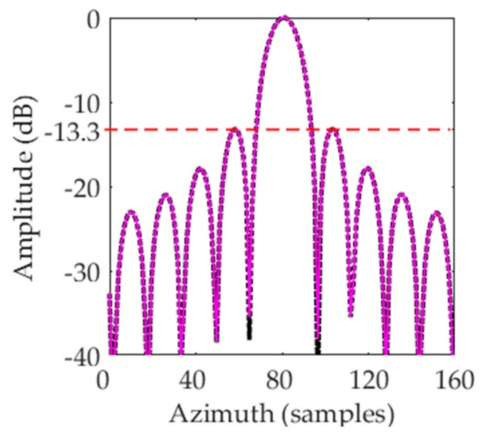

(b)

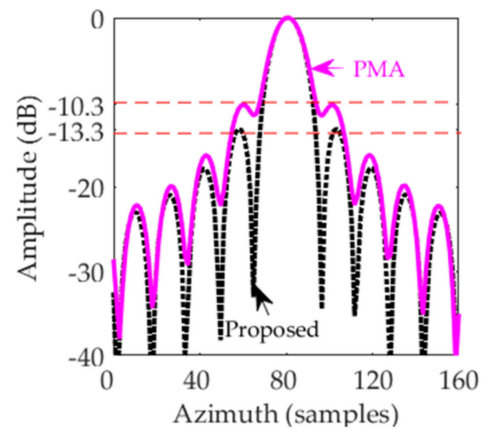

(c)

Figure 10. Simulation results: (a) comparative results of azimuth profiles of PT1 by PMA and the proposed algorithm; (b) comparative results of azimuth profiles of PT5 by PMA and the proposed algorithm; (c) comparative results of azimuth profiles of PT9 by PMA and the proposed algorithm.

Table 3. Imaging quality parameters in Simulation Case II.

\begin{tabular}{ccccc}
\hline Method & Target & IRW $(\mathbf{m})$ & PSLR (dB) & ISLR (dB) \\
\hline \multirow{3}{*}{ Proposed algorithm } & PT1 & 0.474 & -13.27 & -10.07 \\
& PT5 & 0.472 & -13.31 & -10.04 \\
& PT9 & 0.471 & -13.30 & -10.01 \\
\hline \multirow{3}{*}{ PMA } & PT1 & 0.613 & -4.93 & -4.64 \\
& PT5 & 0.423 & -13.32 & -10.06 \\
& PT9 & 0.546 & -10.35 & -7.58 \\
\hline
\end{tabular}

\subsection{Scene Simulation Results}

Corresponding to the experiment of the spot matrix simulation, the scene simulation results were presented in this subsection. The echo data of the scene was obtained by the time-domain simulation method, with motion errors as shown in Figure 9a. The imaging results of the echo data validated the efficiency of the proposed EBP algorithm. Here, it was assumed that the data of an X-band SAR with the squinted spotlight mode was used to demonstrate the performance of the proposed algorithm. The center squint angle was about $60^{\circ}$, and the velocity was about $120 \mathrm{~m} / \mathrm{s}$. The whole scene was about $1.2 \mathrm{~km}$ in the cross-range, and $1.5 \mathrm{~km}$ in the range. Figure 11 showed the focusing results, which were processed by traditional BPA, the proposed algorithm, and PMA, respectively. Particularly, the topography variations were added into the dotted line rectangular region in Figure 11. The difference between the highest and the lowest points in the topography variations was $310.36 \mathrm{~m}$.

The entire scene was well focused, with the proposed algorithm in Figure 11b, including the edge regions. In order to obtain a clear and detailed comparison, the local enlargements of the solid line rectangular regions of each image were displayed respectively in the dashed line rectangular regions. It was evident that the imaging quality in Figure $11 \mathrm{~b}$ was as good as the one in Figure $11 \mathrm{a}$, and it was better than the one in Figure 11c. Therefore, the proposed algorithm could handle the effects of the topography variations well, for high-resolution airborne SAR. 


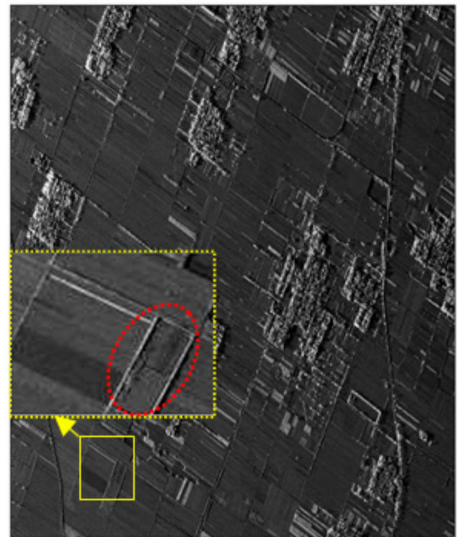

(a)

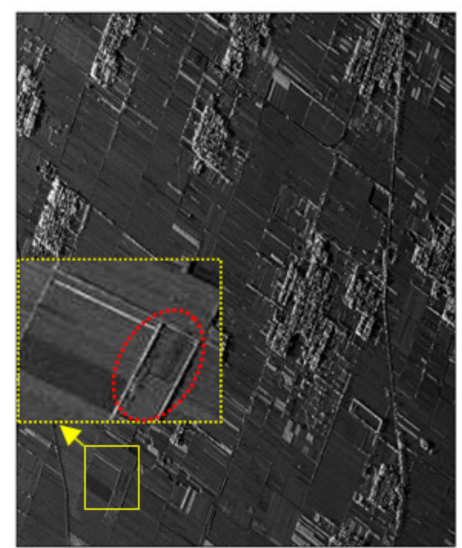

(b)

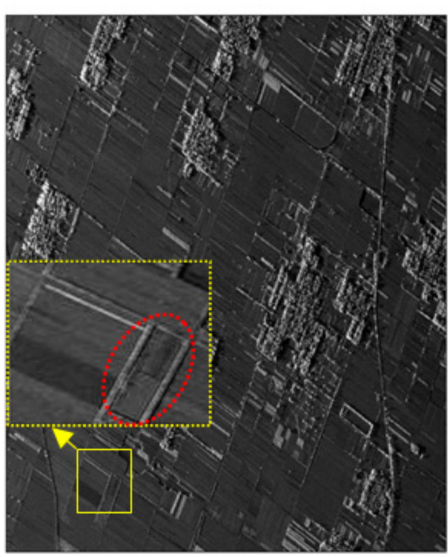

(c)

Figure 11. Scene simulation data results processed by different algorithms. (a) Scene simulation data results processed by traditional BPA. (b) Scene simulation data results processed by the EBP algorithm.

(c) Scene simulation data results processed by the reference algorithm.

\section{Discussion}

\subsection{Parameter Selection}

In the Section 3.1, the echo signal may have aliasing after the frequency-domain processing, if the scaling factor $\alpha_{0}$ was selected improperly. To weaken the signal aliasing, the zero padding operation was taken in some literatures. However, the zero padding would increase the computation burden of the whole algorithm, which was the opposite of the purpose of the proposed algorithm. Therefore, proper selection for the parameter $\alpha_{0}$ without zero padding operation was necessary for frequency-domain processing. As has been discussed in [51], the two-step processing could be regarded as a special case of two Fractional Fourier transform (FrFT) operations. Similarly, the frequency-domain processing could also be implemented by two FrFT operations with parameters, $\chi$ and $\kappa$. We obtained the relation between the parameters as:

$$
\begin{gathered}
\chi+\kappa=\frac{\pi}{2} \\
\cot \chi=-\pi \alpha_{0}
\end{gathered}
$$

After frequency-domain processing, the whole bandwidth of the echo signal should be less than the pulse repetition frequency (PRF), in the case of the phenomenon of frequency aliasing. It means that:

$$
\begin{gathered}
T_{a}\left|\cot \eta_{0}+\tan \chi\right|+2 \pi B_{s} \leq 2 \pi P R F \\
T_{a}\left|\cot \eta_{0}\right|+2 \pi B_{s} \leq \frac{m^{\prime}}{m} T_{a}|\tan \chi|
\end{gathered}
$$

where $\eta_{0}$ is the original slant angle of a point target in the time-frequency distribution, $B_{S}$ is the instantaneous bandwidth of the echo signal in the receiving terminal, $m$ and $m^{\prime}$ denote the numbers of sampling points in the original azimuth time-domain and the new azimuth time-domain, respectively.

The selection of parameter $\alpha_{0}$ is usually obtained through the Equations (14)-(17). In general, the parameter should be selected, with the guarantee that the supporting area of the rotated signal should be in its available area.

\subsection{Computational Burden}

It is well-known that the BPA can precisely manage the problems caused by the curved path model. However, the BPA has a much heavier computational burden than that of the frequency-domain algorithms. In essence, the proposed algorithm in this paper was an improved BP algorithm, so it 
was necessary to make discussion about the computational burden of the proposed algorithm here. For simplification and without loss of generality, the computational burden of the traditional CS was compared as the standard.

The frequency-domain processing in this work was essentially a frequency-domain algorithm. In the frequency-domain processing, three azimuth fast Fourier transforms (FFTs), and three multiplications are utilized. Therefore, the total computational burden of the frequency-domain processing could be expressed as:

$$
O_{F}=1.5 N M \log _{2} M+3 N M
$$

where $M$ is the number of the azimuth samples, and $N$ is the number of the range samples. Moreover, the computational burden of time-domain processing presented in Section 3.2 is discussed. Considering the focusing algorithm, i.e., two range FFTs, one multiplication, and BPA, the computational burden of the time-domain processing can be derived as:

$$
\mathrm{O}_{T}=M N+N M \log _{2} N+\mu_{\mathrm{int}} M^{2} N
$$

where $\mu_{\text {int }}$ denotes the proportional factor of the computational burden of the post-processing, which is determined by the rotation factor. Thus, with Equations (18) and (19), the total computational burden of the EBP algorithm was expressed as:

$$
\mathrm{O}_{s}=1.5 N M \log _{2} M+4 N M+N M \log _{2} N+\mu_{\text {int }} M^{2} N
$$

In comparison, of the BPA (e.g., [52]), for each pixel in the final image, a signal vector with a length $M$ was extracted from the range-compressed data, multiplied with a phase function, and summed; thus, the total computational burden could be expressed as:

$$
O_{B}=M^{2} N
$$

In a standard CSA, two range FFTs, one azimuth FFT, and three multiplications are used. Therefore, the computational burden of the standard CSA can be expressed as:

$$
\mathrm{O}_{\mathrm{C}}=\mathrm{MN} \log _{2} \mathrm{~N}+\mathrm{NM} \log _{2} \mathrm{M}+3 N M
$$

The results of the comparison mentioned above with specific parameters, are shown in Table 4, based on Equations (20)-(22). As listed in Table 4, ratios at five sample sizes were computed to quantify the comparison. Clearly, the computational burden of the BPA was much larger than that of the proposed algorithm, which meant that our method could greatly improve the operation speed. Even the computational burden of the proposed algorithm was slightly larger than that of the CSA, but there was wide application in the high-resolution airborne SAR, with topography variations.

Table 4. Computational load analysis using CSA as references.

\begin{tabular}{cccccc}
\hline Data Size in Azimuth $\left(\times \mathbf{1 0}^{\mathbf{3}}\right)$ & $\mathbf{1}$ & $\mathbf{2}$ & $\mathbf{4}$ & $\mathbf{8}$ & $\mathbf{1 6}$ \\
\hline BPA/CSA & 150.30 & 290.11 & 560.73 & 1084.92 & 2101.48 \\
Proposed/CSA & 12.07 & 22.15 & 41.64 & 79.39 & 152.59 \\
\hline
\end{tabular}

\section{Conclusions}

The requirement for the resolution for remote sensing has become higher and higher. When the resolution is at the decimeter level, topography variations were ignored in conventional airborne SAR would cause serious phase errors. For the high-resolution airborne SARs with topography variations, 3-D variations in time or frequency-domains were difficult to eliminate with the traditional 
algorithms. Especially for the topography variations, the expression of the received echo data in the frequency-domain was difficult to derive. In this paper, based on the precise physical model of airborne SAR considering the topography variations, phase errors caused by topography variations are analyzed in different domains first.

The proposed EBP algorithm includes two main parts: frequency-domain processing and time-domain processing. The frequency-domain processing is essentially an operation of timefrequency rotation to reduce the virtual aperture length greatly, by selecting a suitable rotation factor. The computational burden can be decreased by removing the redundant position in the flight track. Then the accurate range history is obtained with DEM data in the time-domain processing. The BP algorithm can be applied directly in the 2-D time-domain with an accurate range history. In the simulation, the spot matrix simulation results and the scene simulation data validated the performance of the EBP algorithm. Finally, the computational burden of EBP algorithm was compared with those of other algorithms. It was obvious that our algorithm can greatly improve the operation speed compared with the traditional BPA.

Author Contributions: C.L. and S.T. conceived the main idea; S.T., L.Z., and P.G. conceived and designed the experiments; C.L. and S.T. analyzed the data and wrote the paper.

Acknowledgments: This work was supported in part by the National Natural Science Foundation of China under Grants 61601343, 61701393, 61731023, and 61671361, in part by the China Postdoctoral Science Foundation Funded Project under Grant 2016M600768, in part by the National Natural Science Foundation of the Shaanxi Province under grant 2018JM6054, and in part by the National Defense Foundation of China.

Conflicts of Interest: The authors declare no conflict of interest.

\section{References}

1. Cumming, I.G.; Wong, F.H. Digital Processing of Synthetic Aperture Radar Data: Algorithm and Implementation; Artech House: Norwood, MA, USA, 2005.

2. Sun, H.; Shimada, M.; Xu, F.; Xu, F. Recent Advances in Synthetic Aperture Radar Remote Sensing-Systems, Data Processing, and Applications. IEEE Geosci. Remote Sens. Lett. 2017, 14, 2013-2016. [CrossRef]

3. Carrara, W.G.; Majewski, R.M.; Goodman, R.S. Spotlight Synthetic Aperture Radar: Signal Processing Algorithms; Artech House: Norwood, MA, USA, 1995.

4. Dudczyk, J.; Kawalec, A. Optimizing the Minimum Cost Flow Algorithm for the Phase Unwrapping Process in SAR Radar. Bull. Pol. Acad. Sci. Tech. Sci. 2014, 62, 511-516. [CrossRef]

5. Matuszewski, J. The Analysis of Modern Radar Signals Parameters in Electronic Intelligence System. In Proceedings of the 13th International Conference on Modern Problems of Radio Engineering, Telecommunications and Computer Science (TCSET), Lviv, Ukraine, 23-26 February 2016; pp. $298-302$.

6. Matuszewski, J.; Paradowski, L. The Knowledge Based Approach for Emitter Identification. In Proceedings of the 12th International Conference on Microwaves and Radar, Krakow, Poland, 20-22 May 1998; pp. 810-814.

7. Soumekh, M. Synthetic Aperture Radar Signal Processing with MATLAB Algorithms; Wiley-Interscience: Hoboken, NJ, USA, 1999.

8. Zhang, T.; Ding, Z.; Tian, W.; Zeng, T. A 2-D nonlinear chirp scaling algorithm for high squint GEO SAR imaging based on optimal azimuth polynomial compensation. IEEE J. Sel. Top. Appl. Earth Observat. Remote Sens. 2017, 10, 5724-5735. [CrossRef]

9. Tang, S.; Zhang, L.; Guo, P.; Liu, G.; Zhang, Y.; Li, Q.; Gu, Y.; Lin, C. Processing of monostatic SAR data with general configurations. IEEE Trans. Geosci. Remote Sens. 2015, 53, 6529-6546.

10. Sun, G.C.; Wu, Y.; Wang, Y.; Xing, M.D.; Bao, Z. Full-aperture focusing of very high resolution spaceborne-squinted sliding spotlight SAR data. IEEE Trans. Geosci. Remote Sens. 2017, 55, 3309-3321. [CrossRef]

11. Hobbs, S.; Mitchell, C.; Forte, B.; Holley, R.; Snapir, B.; Whittaker, P. System design for geosynchronous synthetic aperture radar missions. IEEE Trans. Geosci. Remote Sens. 2014, 52, 7750-7763. [CrossRef]

12. Wang, C.; Chen, L.; Liu, L. A New Analytical Model to Study the Ionospheric Effects on VHF/UHF Wideband SAR Imaging. IEEE Trans. Geosci. Remote Sens. 2017, 55, 4545-4557. [CrossRef] 
13. Moreira, A.; Krieger, G.; Hajnsek, I.; Papathanassiou, K.P.; Younis, M.; Lopez-Dekker, P.; Huber, S.; Villano, M.; Pardini, M.; Eineder, M.; et al. Tandem-L: A highly innovative bistatic SAR mission for global observation of dynamic processes on the Earth's surface. IEEE Geosci. Remote Sens. Mag. 2015, 3, 8-23. [CrossRef]

14. Fornaro, G.; Franceschetti, G.; Perna, S. Motion Compensation Errors: Effects on the accuracy of airborne SAR Images. IEEE Trans. Aerosp. Electron. Syst. 2005, 41, 1338-1352. [CrossRef]

15. Raney, R.K.; Runge, H.; Bamler, R.; Cumming, I.G.; Wong, F.H. Precision SAR processing using chirp scaling. IEEE Trans. Geosci. Remote Sens. 1994, 32, 786-799. [CrossRef]

16. Cafforio, C.; Prati, C.; Rocca, E.C. SAR focusing using seismic migration techniques. IEEE Trans. Aerosp. Electron. Syst. 1991, 27, 194-207. [CrossRef]

17. Sun, G.C.; Jiang, X.; Xing, M.D.; Qiao, Z.J.; Wu, Y.; Bao, Z. Focus improvement of highly squinted data based on azimuth nonlinear scaling. IEEE Trans. Geosci. Remote Sens. 2011, 49, 2308-2322. [CrossRef]

18. Wong, F.W.; Yeo, T.S. New applications of nonlinear chirp scaling in SAR data processing. IEEE Trans. Geosci. Remote Sens. 2001, 39, 946-953. [CrossRef]

19. Zeng, T.; Hu, C.; Wu, L.; Liu, L.; Tian, W.; Zhu, M.; Long, T. Extended NLCS algorithm of BiSAR systems with a squinted transmitter and a fixed receiver: Theory and experimental confirmation. IEEE Trans. Geosci. Remote Sens. 2013, 51, 5019-5030. [CrossRef]

20. Tang, S.; Zhang, L.; So, H.C. Focusing high-resolution highly-squinted airborne SAR data with maneuvers. Remote Sens. 2018, 10, 862. [CrossRef]

21. Qiu, X.; Hu, D.; Ding, C. An improved NLCS algorithm with capability analysis for one-stationary BiSAR. IEEE Trans. Geosci. Remote Sens. 2008, 46, 3179-3186. [CrossRef]

22. Wang, Y.; Li, J.; Yang, J. Wide Nonlinear chirp scaling algorithm for spaceborne stripmap range sweep SAR imaging. IEEE Trans. Geosci. Remote Sens. 2017, 55, 6922-6936. [CrossRef]

23. Sun, G.C.; Xing, M.D.; Wu, Y.; Wang, Y.; Yang, J.; Bao, Z. A 2-D space-variant chirp scaling algorithm based on the RCM equalization and subband synthesis to process geosynchronous SAR data. IEEE Trans. Geosci. Remote Sens. 2014, 52, 4868-4880.

24. Li, D.; Lin, H.; Liu, H.; Liao, G.; Tan, X. Subaperture approach based on azimuth-dependent range cell migration correction and azimuth focusing parameter equalization for maneuvering high-squintmode SAR. IEEE Trans. Geosci. Remote Sens. 2017, 53, 6718-6734.

25. Macedo, K.A.C.; Scheiber, R. Precise topography- and aperture-dependent motion compensation for airborne SAR. IEEE Trans. Geosci. Remote Sens. 2005, 2, 172-176.

26. Prats, P.; Reigber, A.; Mallorqui, J.J. Topography-dependent motion compensation for repeat-pass interferometric SAR systems. IEEE Trans. Geosci. Remote Sens. 2005, 2, 206-210. [CrossRef]

27. Yang, Z.M.; Sun, G.C.; Xing, M.D. A new fast back-projection algorithm using polar format algorithm. In Proceedings of the Asia-Pacific Conference on Synthetic Aperture Radar (APSAR), Tsukuba, Japan, 23-27 September 2013; pp. 373-376.

28. Rodriguez-Cassola, M.; Prats, P.; Krieger, G.; Moreira, A. Efficient time-domain image formation with precise topography accommodation for general bistatic SAR configurations. IEEE Trans. Aerosp. Electron. Syst. 2011, 47, 2949-2966. [CrossRef]

29. Gazdag, J.; Sguazzero, P. Migration of Seismic Data. Proc. IEEE. 1984, 72, 1302-1315. [CrossRef]

30. Seger, O.; Herberthson, M.; Hellsten, H. Real-time SAR processsing of low frequency ultra wideband radar data. In Proceedings of the EUSAR, Friedrichshafen, Germany, 25-27 May 1998.

31. Ferretti, A.; Prati, C.; Rocca, F. Multibaseline InSAR DEM reconstruction: The wavelet approach. IEEE Trans. Geosci. Remote Sens. 1999, 37, 705-715. [CrossRef]

32. Ferraiuolo, G.; Meglio, F.; Pascazio, V.; Schirinzi, G. DEM reconstruction accuracy in multichannel SAR interferometry. IEEE Trans. Geosci. Remote Sens. 2009, 47, 191-201. [CrossRef]

33. Shao, Y.F.; Wang, R.; Deng, Y.K.; Liu, Y.; Chen, R.; Liu, G.; Balz, T.; Loffeld, O. Digital elevation model reconstruction in multichannel spaceborne/stationary SAR interferometry. IEEE Geosci. Remote Sens. Lett. 2014, 11, 2080-2084. [CrossRef]

34. Nilsson, S. Application of Fast Backprojection Techniques for Some Inverse Problems in Integral Geometry. Ph.D. Thesis 499, Linkoping University, Linkoping, Sweden, 1997.

35. Ulander, L.; Hellsten, H.; Stenström, G. Synthetic-aperture radar processing using fast factorized back-projection. IEEE Trans. Aerosp. Electron. Syst. 2003, 39, 760-776. [CrossRef] 
36. Cao, N.; Lee, H.; Zaugg, E.; Shrestha, R.; Carter, W.; Glennie, C.; Wang, G.; Lu, Z.; Fernandez-Diaz, J.C. Airborne DInSAR results using time-domain backprojection algorithm: A case study over the slumgullion landslide in Colorado with validation using spaceborne SAR, airborne LiDAR, and ground-based observations. IEEE J. Sel. Topics Appl. Earth Observ. Remote Sens. 2017, 108, 4987-5000. [CrossRef]

37. Heng, Z.; Jiangwen, T.; Robert, W.; Yunkai, D.; Wei, W.; Ning, L. An accelerated backprojection algorithm for monostatic and bistatic SAR processing. Remote Sens. 2018, 10, 140. [CrossRef]

38. Tang, J.; Deng, Y.; Wang, R.; Zhao, S.; Li, N.; Wang, W. A weighted backprojection algorithm for azimuth multichannel SAR imaging. IEEE Geosci. Remote Sens. Lett. 2016, 13, 1265-1269. [CrossRef]

39. Yang, L.; Zhou, S.; Zhao, L.; Xing, M. Coherent Auto-Calibration of APE and NsRCM under Fast Back-Projection Image Formation for Airborne SAR Imaging in Highly-Squint Angle. Remote Sens. 2018, 10, 321. [CrossRef]

40. Vu, V.T.; Sjogren, T.K.; Pettersson, M.I. Fast time-domain algorithms for UWB bistatic SAR processing. IEEE Trans. Aerosp. Electron. Syst. 2013, 49, 1982-1994. [CrossRef]

41. Walterscheid, I.; Espeter, T.; Brenner, A.R.; Klare, J.; Ender, J.H.G.; Nies, H.; Wang, R.; Loffeld, O. Bistatic SAR experiments with PAMIR and TerraSAR-x; setup, processing, and image results. IEEE Trans. Geosci. Remote Sens. 2010, 48, 3268-3279. [CrossRef]

42. Shao, Y.; Wang, R.; Deng, Y.; Liu, Y.; Chen, R.; Liu, G.; Loffeld, O. Fast backprojection algorithm for bistatic SAR imaging. IEEE Geosci. Remote Sens. Lett. 2013, 10, 1080-1084. [CrossRef]

43. Duque, S.; Lopez-Dekker, P.; Mallorqui, J.J. Single-pass bistatic SAR interferometry using fixed-receiver configurations: Theory and experimental validation. IEEE Trans. Geosci. Remote Sens. 2010, 48, 2740-2749. [CrossRef]

44. Peng, X.; Wang, Y.; Hong, W.; Wu, Y. Autonomous Navigation Airborne Forward-Looking SAR High Precision Imaging with Combination of Pseudo-Polar Formatting and Overlapped Sub-Aperture Algorithm. Remote Sens. 2013, 11, 6063-6078. [CrossRef]

45. Xu, W.; Deng, Y.; Huang, P.; Wang, R. Full-aperture SAR data focusing in the spaceborne squinted sliding-spotlight mode. IEEE Trans. Geosci. Remote Sens. 2014, 52, 4596-4607. [CrossRef]

46. Tang, S.; Zhang, L.; Guo, P.; Liu, G.; Sun, G.C. Acceleration model analyses and imaging algorithm for highly squinted airborne spotlight mode SAR with maneuvers. IEEE J. Sel. Topics Appl. Earth Observ. Remote Sens. 2015, 8, 1120-1131. [CrossRef]

47. Lanari, R.; Tesauro, M.; Sansosti, E.; Fornaro, G. Spotlight SAR data focusing based on a two-step processing approach. IEEE Trans. Geosci. Remote Sens. 2001, 39, 1993-2004. [CrossRef]

48. Yang, M.D.; Zhu, D.Y.; Song, W. Comparison of two-step and one-step motion compensation algorithms for airborne synthetic aperture radar. Electron. Lett. 2015, 51, 1108-1110. [CrossRef]

49. Lanari, R.; Zoffoli, S.; Sansosti, E.; Fornaro, G.; Serafino, F. New approach for hybrid strip-map/spotlight SAR data focusing. Proc. Inst. Elect. Eng.-Radar Sonar Navig. 2001, 148, 363-372. [CrossRef]

50. Sun, G.C.; Jiang, X.; Xing, M.D.; Xia, X.G.; Wu, Y.R.; Bao, Z. Beam steering SAR data processing by a generalized PFA. IEEE Trans. Geosci. Remote Sens. 2013, 51, 4366-4377. [CrossRef]

51. Sun, G.C.; Xing, M.D.; Xia, X.G.; Yang, J.; Wu, Y.R.; Bao, Z. A unified focusing algorithm for several modes of SAR based on FrFT. IEEE Trans. Geosci. Remote Sens. 2013, 51, 3139-3155. [CrossRef]

52. Xie, H.; Shi, S.; An, D.; Wang, G.; Wang, G.; Xiao, H.; Huang, X.; Zhou, Z.; Xie, C.; Wang, F.; et al. Fast factorized backprojection algorithm for one-stationary bistatic spotlight circular SAR image formation. IEEE J. Sel. Top. Appl. Earth Observ. Remote Sens. 2017, 10, 1494-1510. [CrossRef]

(C) 2018 by the authors. Licensee MDPI, Basel, Switzerland. This article is an open access article distributed under the terms and conditions of the Creative Commons Attribution (CC BY) license (http://creativecommons.org/licenses/by/4.0/). 